\title{
Seroprevalencia de micosis pulmonares en pacientes con sintomatología de enfermedad respiratoria crónica baja. Alto Huallaga, Perú 2004
}

\author{
Walter Gómez ${ }^{1}$, Miriam Guevara ${ }^{2}$, Carlos Cortegana ${ }^{1}$, Pedro Obregon ${ }^{3}$, \\ Juan Motta ${ }^{1}$, Nancy Antholveg ${ }^{1}$
}

Resumen

Objetivo: Detectar por serología la presencia de micosis de localización pulmonar (Paracoccidioides brasiliensis, Aspergillus sp. e Histoplasma capsulatum), en pacientes con síntomas de enfermedad respiratoria crónica. Diseño: Estudio transversal. Lugar: Alto Huallaga (selva de los departamentos de Huánuco y San Martín), Perú. Pacientes: Se incluyó personas procedentes de seis distritos (Rupa Rupa, Tocache, Uchiza, Santa Lucia, Aucayacu y Puerto Inca - Sungaro) con diagnóstico de enfermedad respiratoria crónica baja, tres episodios o más de bronquitis al año y/o tos con expectoración por más de tres meses y en quienes se descartó tuberculosis pulmonar mediante baciloscopia directa. Intervenciones: Por cada persona se llenó una ficha clínica-epidemiológica durante abril a septiembre de 2004, seguido de la toma de una muestra de suero $(10 \mathrm{~mL})$ para las pruebas de inmunodifusión para Histoplasma capsulatum, Paraccocidiodes brasiliensis, Aspergilus sp, y Aspergillus fumigatus. Principales medidas de resultados: Seroprevalencia de micosis a Histoplasma capsulatum, Aspergilosis spp. $y$ Paracoccidioides brasiliensis. Resultados: De 640 casos, 327 (51,1\%) fueron de sexo masculino, edad promedio 37,1 años y tiempo de enfermedad de 7,4 meses. La seroprevalencia de micosis fue 1,7\% (11/640): 4 Histoplasma capsulatum y 7 Aspergilosis spp. No se obtuvo casos positivos de Paracoccidioides brasiliensis y, en los casos positivos, solo se encontró asociación con 'baja de peso' ( $p=0,004)$. Conclusión: Si bien la frecuencia micoserológica obtenida fue baja, se sugiere en esta zona endémica considerar a las micosis dentro del diagnóstico diferencial de casos con enfermedades respiratorias crónicas bajas, así como la utilización de técnicas de inmunodifusión para el diagnóstico de estas etiologías.

Palabrasclave Neumopatías fungicas; enfermedades respiratorias; inmunodifusión; Perú, enfermedades respiratorias.

Pulmonary mycosis seroprevalence in patients with chronic low respiratory symptomatology. Alto Huallaga, Peru 2004

\begin{abstract}
Objective: To detect lung mycosis (Paracoccidioides brasiliensis, Aspergillus sp., and Histoplasma capsulatum) with chronic respiratory disease symptoms (Huanuco and San Martin departments forest). Design: Cross-sectional study. Setting: Peru's Alto Huallaga. Patients: Study done in six districts' (Rupa Rupa, Tocache, Uchiza, Santa Lucia, Aucayacu and Puerto IncaSungaro) subjects with diagnosis of chronic respiratory disease,
\end{abstract}

\footnotetext{
1 Hospital Tingo María - EsSalud. Huanuco, Perú.

2 Laboratorio de Micología, Instituto Nacional de Salud. Lima, Perú.

3 Hospital Huanuco - Essalud. Huanuco, Perú.
}

three or more episodes of bronchitis per year and/or cough for over three months in whom pulmonary tuberculosis was screened by direct baciloscopy. Interventions: Each subject had a clinical epidemiological card filled and $10 \mathrm{~mL}$ of blood was collected for immunodiffusion test for Histoplasma capsulatum, Paraccocidiodes brasiliensis, and Aspergilus sp, between April and September 2004. Main outcome mesasures: Mycosis seroprevalence, including Histoplasma capsulatum, Aspergilus spp., and Paracoccidioides brasiliensis Results: From 640 cases, 327 (51,1\%) were males, avegare age 37,1 years and time of disease 7,4 months. Mycosis seroprevalence was 1,7\% (11/640), including 4 Histoplasma capsulatum and 7 Aspergilus spp. No positive cases of Paracoccidioides brasiliensis was obtained and the positive cases were associated only to 'low weight' $(p=0,004)$. Conclusion: Although the mycosis serologic frequency was low, we suggest to consider mycosis in the differential diagnosis of 
cases with chronic respiratory diseases in endemic zones, as well as the use of immunodiffusion techniques for diagnosis.

Key words: Lung diseases, fungal; respiratory tract diseases; immunodiffusion; Peru, respiratory tract diseases.

\section{INTRODUCCIÓN}

Las enfermedades broncopulmonares constituyen un importante problema de salud pública, sobre todo en aquellos países donde la incidencia de tuberculosis pulmonar es alta o presentan zonas endémicas para las micosis; ello, por la semejanza del cuadro clínico o por su asociación de la última a los cuadros de tuberculosis $\left({ }^{1}\right)$.

En este tipo de infecciones respiratorias, participan principalmente dos tipos de agentes fúngicos: los hongos dimórficos Histoplasma capsulatum y Paracoccidioides brasiliensis- y los hongos oportunistas, entre los que destacan Aspergillus sp $\left({ }^{2}\right)$.

En el Perú, estas infecciones también han sido identificadas. Diversos reportes han demostrado la presencia de casos en algunas zonas del país $\left({ }^{3-12}\right)$, aunque es poco lo que se conoce sobre su real frecuencia.

Las micosis son causadas por microorganismos fungi que viven libres en la naturaleza. Generalmente, se hallan en cuevas naturales y en el suelo y se limitan a ciertas áreas geográficas ubicadas en cuencas hidrográficas de clima cálido y húmedo; este es el caso de la zona del Alto Huallaga (ubicada al nor-oriente peruano).

La vía de ingreso de estas infecciones fúngicas es a través de la inhalación de esporas del hongo hacia el pulmón, a partir del que puede diseminarse a otros órganos. Por ello, frecuentemente, cursan con síntomas de un cuadro respiratorio que tiende a ser crónico. En algunos casos, dado los síntomas presentados, se confunde con patologías frecuentes, como la tuberculosis pulmonar, o se presentan de forma sobreagregada a esta enfermedad, dado que los hongos pueden alojarse en cavidades 'naturales' (lesiones post-secuela de enfermedad) de las personas.

En la zona del Alto Huallaga, que comprende provincias de los departamentos de Huánuco y San Martín, es frecuente el reporte de esta enfermedad, sobre todo en las provincias de Leoncio Prado y Tocache, dado que son zonas con innumerables cuevas de atracción turística (Red EsSalud Tingo María - Huánuco; 1995-2002. Reporte de casos de micosis pulmonar. Información no publicada).

Dado que en nuestra observación es frecuente la presencia de personas con síntomas de enfermedades respiratorias crónicas bajas, que continuamente acuden a los establecimientos de salud de la zona, sin llegar a establecerse en muchos casos el agente causal específico de la sintomatología presentada, es importante descartar la presencia de una enfermedad micótica pulmonar.

Para ello, idealmente, se debería realizar el aislamiento del hongo en muestras procedentes de vías pulmonares, que inicialmente incluye la obtención y siembra de muestras seriadas de esputo (generalmente difícil de conseguir y mantener en condiciones óptimas para el cultivo micológico); aunque, con el fin de incrementar la eficiencia diagnóstica, puede llegarse a realizar técnicas invasivas a los pacientes (lo que requiere personal y equipos especializados). Sin embargo, debido a las dificultades que conllevan dichos procedimientos, se ha desarrollado alternativas diagnósticas, que incluyen la detección de anticuerpos específicos en el suero de los pacientes, como indicador de alta sospecha de micosis activa, siendo muchas veces éste el único hallazgo de laboratorio.

La necesidad de incrementar significativamente la cobertura diagnóstica de las micosis pulmonares, que permitan tratamientos oportunos y eviten sus secue- 
las (reemplazo de tejido funcional por tejido cicatricial), convierten los métodos serológicos en herramientas útiles $\left.{ }^{(13}\right)$.

Las pruebas cutáneas con histoplasmina y paracoccidioidina son usadas para determinar la endemia de estas patologías y solo permiten revelar el contacto previo con el hongo y no diagnostican la enfermedad $\left({ }^{14,15}\right)$.

En tanto, el serodiagnóstico por inmunodifusión es un método que en los últimos años ha tomado gran importancia, sobre todo para el diagnóstico de micosis provocadas por agente dimorfos (el mejoramiento en la calidad de los antígenos, convirtió a esta en una metodología reproducible y específica para la investigación de patologías fúngicas en población inmunocompetente) $\left({ }^{13,16-18}\right)$. Este método alcanza una especificidad superior al $90 \%$ para el diagnóstico de micosis endémicas en población inmunocompetente. Así como en el caso de la aspergilosis, su certeza diagnóstica depende de los antígenos utilizados y de la forma clínica de la enfermedad (la sensibilidad del método varía entre $50 \%$ en aspergilosis broncopulmonar alérgica y $70 \%$ en aspergiloma) $\left({ }^{19,20}\right)$.

En tal sentido, se planteó la presente investigación, buscando evaluar serológicamente la presencia de tres tipos de micosis pulmonar (paracoccidioidomicosis, aspergilosis e histoplasmosis), en pacientes con síntomas de enfermedad respiratoria crónica residentes de la zona del Alto Huallaga, Perú. Esta investigación nos podrá servir como referencia para entender la magnitud de la enfermedad en la zona, los factores asociados a su presencia, así como la utilidad del método diagnóstico.

\section{MATERIALES Y MÉTODOS}

Se realizó un estudio observacional, descriptivo y transversal, entre los meses de abril y septiembre del año 2004, en la zona del Alto Huallaga, comprendiendo las provincias de Leoncio Prado y Tocache (seis distritos: Rupa Rupa, Tocache, Uchiza, Santa Lucia, Aucayacu y Puerto Inca Sungaro). Se escogió estas zonas tropicales por ser consideradas endémicas para las micosis en estudio.

Empleando el programa estadístico Statcal de Epi Info v.6, se calculó el tamaño muestral. Para ello, se consideró una población de 10422 (número de pacientes con enfermedades respiratorias crónicas bajas atendidas en el año 2002), un $p$ de $20 \%$, un error esperado de $3 \%$ y un nivel de confianza del $95 \%$, obteniéndose un tamaño muestral de 640 pacientes. Luego de ello, se realizó una distribución del aporte muestral de cada establecimiento de salud, teniendo como referencia el número de casos presentados el año 2002. La selección de los casos se realizó mediante un muestreo no probabilístico, siendo esta de forma consecutiva, hasta completar el tamaño muestral asignado a cada establecimiento. Se incluyó a todas las personas mayores de 18 años que acudieron a los establecimientos de salud seleccionados y que cumplieron con la 'definición de caso' (pacientes con diagnóstico de enfermedad respiratoria crónica baja, con tres episodios a más de bronquitis al año y/o tos con expectoración por más de tres meses y que presentara descarte de tuberculosis pulmonar mediante baciloscopia directa). Se excluyó aquellos casos con diagnóstico de tuberculosis pulmonar activa o con diagnóstico de otra etiología confirmada causante de una enfermedad respiratoria crónica baja diferente a la del estudio.

La captación de los casos se realizó en el servicio de consultorios externos de los establecimientos de salud del Ministerio de Salud y de EsSalud, de los distritos de Rupa Rupa, Tocache, Uchiza, Santa Lucía, Aucayacu y Puerto Inca - Súngaro. A las personas que aceptaron participar en el estudio, se procedió a obtener datos que fue- 
ron recogidos en una ficha clínicaepidemiológica (que también incluyó la obtención de factores asociados a la presencia de las enfermedades en estudio), a cargo del personal médico del establecimiento de salud previamente capacitado en su llenado, seguido de la toma de una muestra de sangre $(10 \mathrm{~mL})$, para las pruebas de micoserología. Todos los sueros fueron conservados y derivados -a temperatura de $4^{\circ}$ a $10{ }^{\circ} \mathrm{C}$, empleando bloques de hielo- al Laboratorio de Micología del Instituto Nacional de Salud (INS) de Lima, utilizando los mecanismos de la Red de Laboratorios de Salud Pública del país. El envío de las muestras se realizó de forma semanal. En el INS se realizó las pruebas de inmunodifusión en gel agar para Histoplasma capsulatum (código interno 207021003), Paraccocidiodes brasiliensis (código interno 207021103), Aspergilus sp (código interno 207020703) ${ }^{(21)}$. Los reactivos utilizados fueron antígenos monovalentes, con determinantes antigénicos específicos para Histoplasma capsulatum, Paraccocidiodes brasiliensis, Aspergilus sp.

Este estudio contó con la aprobación del Comité de Ética en Investigación del Instituto Nacional de Salud, obteniéndose previo a la inclusión de los participantes su consentimiento informado escrito, actividad que estuvo a cargo de los responsables del estudio en cada establecimiento. Además, se les hizo entrega de los resultados de laboratorio a los participantes en forma mensual, derivándose los casos positivos a los establecimientos de salud respectivos, para su manejo.

Para el análisis estadístico, se realizó un análisis univariado y bivariado, utilizando la prueba chi cuadrado y la prueba exacta de Fisher, según correspondiese. Se consideró un $p<0,05$ como significativo. En el procesamiento y análisis de los datos, se utilizó el programa SPSS 11,0 para windows.

\section{RESULTADOS}

Un total de 640 casos ingresaron al estudio (395 de establecimientos del Ministerio de Salud y 245 de establecimientos de EsSalud), $327(51,1 \%)$ de sexo masculino, con una edad promedio de 37,1 años (rango de 18 a 75 años), y un tiempo promedio de enfermedad de 7,4 meses (rango de 3 a 24 meses). Las características clínicas epidemiológicas de los pacientes incluidos, se describe en la Tabla 1.

Tabla 1. Características clínicas - epidemiológicas de los 640 pacientes con diagnóstico de enfermedad respiratoria crónica baja procedentes de zonas del Alto Huallaga, 2004.

\begin{tabular}{|c|c|c|}
\hline Característica & Frecuencia & $\%$ \\
\hline \multicolumn{3}{|l|}{ Sexo } \\
\hline Masculino & 327 & 51,1 \\
\hline Femenino & 313 & 48,9 \\
\hline \multicolumn{3}{|l|}{ Edad } \\
\hline 18 a 24 años & 195 & 30,5 \\
\hline 25 a 34 años & 147 & 22,9 \\
\hline 35 a 44 años & 116 & 18,1 \\
\hline 45 a 59 años & 101 & 15,7 \\
\hline 60 a más años & 81 & 12,8 \\
\hline \multicolumn{3}{|l|}{ Procedencia } \\
\hline Rupa rupa (Tingo María) & 187 & 29,2 \\
\hline Aucayacu & 134 & 20,9 \\
\hline Tocache & 95 & 14,8 \\
\hline Uchiza & 89 & 13,9 \\
\hline Puerto Inca - Súngaro & 77 & 12,0 \\
\hline Santa Lucía & 58 & 9,1 \\
\hline \multicolumn{3}{|l|}{ Antecedente patológicos } \\
\hline Bronquiectasias & 103 & 16,1 \\
\hline Asma bronquial & 56 & 8,8 \\
\hline Tuberculosis pulmonar & 55 & 8,6 \\
\hline Virus de inmunodeficiencia humana & 2 & 0,3 \\
\hline \multicolumn{3}{|l|}{ Hábitos de riesgo } \\
\hline Visitar cuevas turísticas & 273 & 42,7 \\
\hline Antecedente de traumatismo con astillas & 85 & 13,3 \\
\hline \multicolumn{3}{|l|}{$\begin{array}{l}\text { Síntomas / signos al momento de la } \\
\text { captación del caso }\end{array}$} \\
\hline Dolor torácico & 419 & 65,5 \\
\hline Baja de peso & 256 & 40,0 \\
\hline Fiebre & 172 & 26,9 \\
\hline Diseña & 128 & 20,0 \\
\hline \multicolumn{3}{|l|}{ Descripción radiológica (en 245 casos) } \\
\hline Presencia de cavidades & 8 & 3,3 \\
\hline Presencia de fibrosis & 7 & 2,9 \\
\hline Derrame pleural & 3 & 1,2 \\
\hline Neumotórax & 4 & 1,6 \\
\hline
\end{tabular}


La seroprevalencia de micosis, en los casos incluidos con sintomatología respiratoria, fue $1,7 \%(11 / 640)$, considerando cualquiera de las tres etiologías estudiadas, encontrándose 4 casos con serología positiva para Histoplasma capsulatum y 7 para Aspergilosis spp. No se obtuvo casos con serología positiva para Paracoccidioides brasiliensis (Tabla 2).

La procedencia de los casos positivos a Histoplasma capsulatum fueron: Rupa rupa 2 casos, Aucayacu 1 caso y Tocache 1 caso; en tanto que, para Aspergilosis spp. fueron: Rupa rupa 6 casos y Puerto Inca Súngaro 1 caso.

El tiempo de enfermedad promedio de los casos que resultaron serológicamente positivos a Histoplasma capsulatum y Aspergilosis spp. fue de 4 y 15,9 meses, respectivamente. Dos de los 11 casos positivos obtuvieron serología positiva, tanto para Histoplasma capsulatum como para Aspergilosis spp., siendo ambos varones y con dolor torácico y baja de peso como síntomas comunes únicos.

En general, de los casos serológicamente positivos solo se encontró asociación con el síntoma 'baja de peso' $(p=0,004)$. Y de forma específica, se encontró una asociación a seropositividad para Histoplasma capsulatum con 'baja de peso' $(p=0,025)$ y seropositividad para Aspergilosis spp. con 'baja de peso' $(p=0,018)$ y dolor torácico

Tabla 2. Serodiagnóstico de micosis pulmonares mediante prueba de inmunodifusión.

\begin{tabular}{lrrrrrr}
\hline \multirow{2}{*}{ Agente } & \multicolumn{4}{c}{ Inmunodifusión } \\
\cline { 2 - 3 } & \multicolumn{3}{c}{ Positivos } & & \multicolumn{3}{c}{ Negativos } \\
\cline { 2 - 3 } & Frecuencia & $\%$ & & Frecuencia & $\%$ \\
\hline Histoplasma capsulatum & 4 & 0,62 & & 636 & 99,38 \\
Aspergilosis spp & 7 & 1,10 & & 633 & 98,90 \\
Paracoccidioides brasiliensis & 0 & 0 & & 640 & 100 \\
Total & 11 & 1,72 & & 629 & 98,28 \\
\hline
\end{tabular}

$(p=0,05)$. Visitar cuevas o antecedente de traumatismo con astillas, así como el resto de variables evaluadas, no tuvieron asociación con los resultados seropositivos.

\section{DISCUSIÓN}

Las micosis profundas constituyen infecciones endémicas en el Perú, así como en muchos países de América y, dado que no son enfermedades de notificación obligatoria, se desconoce su casuística real $\left({ }^{22,23}\right)$.

Estas enfermedades son causadas por microorganismos fungi que viven libres en la naturaleza, en el suelo o en el material orgánico en putrefacción y que con frecuencia están limitados a ciertas regiones geográficas. En dichas zonas, los individuos adquieren esta micosis, aunque la mayoría solo desarrollan síntomas menores o incluso cursan asintomáticos; solo una minoría de los afectados progresan a una enfermedad completamente desarrollada, que puede llegar a ser grave y mortal $\left({ }^{24}\right)$. Quizá esto podría ser una de las explicaciones de la baja frecuencia de casos positivos obtenidos $(1,7 \%)$.

En el caso de Histoplasma capsulatum, este hongo se encuentra preferentemente en suelos ácidos, con alto contenido de nitrógeno y humedad, encontrándose en lugares donde habitan aves -gallineros-, cuevas y agujeros de árboles. La infección se adquiere por inhalación de esporas, requiriéndose para producir infección clínica un alto inóculo.

Nuestro estudio se centró en la búsqueda de micosis pulmonar, dado que este órgano casi siempre se compromete en las micosis profundas, constituyéndose además en el punto de partida para la diseminación a otros órganos $(25,26)$.

Para un adecuado diagnóstico de esta patología, se incluye la combinación de 
cuatro características: cuadro clínicoradiológico, examen microscópico directo y cultivo de especimenes, exámenes histopatológicos y pruebas serológicas. En el caso del cuadro clínico, la literatura menciona síntomas y signos variados, que incluyen tos, expectoración purulenta, dolor torácico, disnea, hemoptisis, fiebre (la mayoría de estos también encontrados en nuestro estudio), llegando incluso algunos casos al shock y la insuficiencia respiratoria $\left({ }^{27}\right)$. Asimismo, además de la agudeza y experiencia clínica, es importante el contexto epidemiológico, que incluye variables como exposición, zona geográfica endémica, factores individuales (ej. inmunosupresión) y compromiso de otros órganos (ejemplo: en el caso de aspergillosis, presencia de sinusitis; antecedentes de TBC (tratadas) y en el caso de blastomicosis, presencia de lesiones dermatológicas) ${ }^{28}$ ). Por ejemplo, en el caso de la aspergiolosis, es frecuente su asociación a la tuberculosis y otras infecciones previas, curadas, pero con secuelas cavitarias, incluyendo otras micosis pulmonares, como la histoplamosis $\left({ }^{27,28}\right)$. Ello se corrobora, en nuestro trabajo, al encontrar coinfección en dos de los casos seropositivos. En el caso de la imagenología, tampoco se encuentra características específicas para las micosis, salvo excepciones, pudiendo encontrarse en el caso de las radiografías de tórax variados cuadros, como lesiones inflamatorias de forma neumónica o bronconeumónica, infiltrados miliares, nódulos, cavitación, calcificación, reacción pleural, adenopatías hiliares; además, la clásica imagen del aspergiloma (fungus ball, micetoma) no siempre está presente en la radiología de aspergilosis pulmonar; así como tampoco, en el caso de histoplasmosis pulmonar, no siempre se aprecia las típicas calcificaciones, ni es tampoco ello la única causa de calcificación en el pulmón ${ }^{(27)}$. En nuestro estudio, no se encontró características radiológicas particulares de los casos seropositivos.
Debido a este cuadro inespecífico, es de suma importancia la ayuda diagnóstica por pruebas de laboratorio, siendo el examen microscópico directo y el cultivo microbiológico los métodos más importantes en el diagnóstico de las infecciones por hongos. Sin embargo, estos métodos requieren personal altamente entrenado para la observación directa del agente en preparaciones microscópicas, así como medidas de bioseguridad adecuadas para el manejo de los aislamientos, dado que las esporas de los hongos dimorfos en fase micelial, son altamente infectivas $\left({ }^{29}\right)$. Además, otro inconveniente es su baja sensibilidad en esputo y las limitaciones de los establecimientos de salud para acceder a un espécimen más conveniente (lavado bronquial, lavado bronquioloalveolar o biopsia) $\left.{ }^{(27,28}\right)$. En nuestro estudio, los establecimientos incluidos en el estudio se encontraban alejados y carecían de los equipos necesarios, por lo que se optó por el transporte de las muestras desde las zonas de recolección hasta la ciudad de Huánuco (al laboratorio de referencia regional) y de ahí a la ciudad de Lima (laboratorios del INS). A pesar de haberse buscado preservar la cadena de frío, estimamos que algunas muestras pueden haber presentado el deterioro del espécimen con su respectivo seronegatividad, situación que podría explicar también nuestros resultados. Además que, en el caso del examen y cultivo de esputo, es importante considerar también la forma de recojo de las muestras, dado que por ejemplo estas muestras no tienen valor si previamente no se realiza un aseo bucal, o si estas no se procesan en las dos primeras horas de recogida la muestra, situación difícil de realizar en algunas zonas del país, donde el apoyo de laboratorio no se encuentra cercano. A ello, hay que agregar que, en el caso del cultivo, para confirmar el diagnóstico micológico se necesita entre 3 a 6 semanas $\left({ }^{29}\right)$.

Debido a lo anterior, es que, para apoyar el diagnóstico micológico, se ha desa- 
rrollado algunos marcadores serológicos confiables, siendo las técnicas más usadas actualmente la determinación de precipitinas (anticuerpos), LPA (antígenos circulantes), fijación de complemento e inmunodifusión. Esta última empleada en el presente estudio.

Discutiendo las características epidemiológicas de los pacientes incluidos, destaca que Rupa rupa (Tingo María) informa la mayor cantidad de casos, ello probablemente relacionado a que esta ciudad ostenta un reconocido atractivo turístico a nivel nacional e internacional, al ser una de las ciudades que está rodeada por la mayor cantidad de cuevas naturales del mundo $\left({ }^{30}\right)$, siendo la más conocida la denominada Cueva de las Lechuzas. Esta cueva es el principal y más visitado atractivo turístico de la zona, habiendo sido también explorada; en cerca de 405 metros de largo, se ha constatado vida en los primeros 265 metros, mayormente habitado por aves como el guacharo (Steatornis caripensis) y Ara $s p .\left({ }^{30}\right)$, y que se comportan como vectores de esporas de Histoplasma capsulatum $\left({ }^{20}\right)$. Debido a que en otros estudios se ha identificado casos de personas con enfermedad micótica, sobre todo relacionado a histoplasmosis, y que presentan el antecedente de haber visitado estas cuevas $\left({ }^{4}\right)$, es necesario que los visitantes sean informados correctamente de las medidas de prevención necesarias y eviten sufrir la afección primaria y sistémica de la denominada 'fiebre de Tingo María'. Si bien nuestros resultados son bajos para la presencia esperada de esta micosis pulmonar (en zonas endémicas de Argentina, se reporta una seropositividad de $11,92 \%)\left({ }^{17}\right)$, creemos que la búsqueda de estas etiologías debe ser considerada en el diagnóstico diferencial de pacientes con enfermedad respiratoria crónica y con sospecha epidemiológica. Si bien, el diagnóstico de certeza de las micosis es la observación del hongo parasitando el tejido del hospedador, la detección de anticuerpos específicos en el sue- ro de los pacientes indica una alta sospecha de micosis activa y muchas veces puede ser el único hallazgo de laboratorio $\left({ }^{17}\right)$.

En la medida de lo posible, lo prudente es englobar los cuatro pilares diagnósticos previamente mencionados (cuadro clínicoepidemiológico, radiológico, examen microscópico directo y cultivos de especimenes y prueba serológica). Se recomienda continuar con estudios analíticos que evalúen diversos factores de riesgo asociados a micosis pulmonares prevalentes en esta zona.

\section{AGRADECIMIENTOS}

Este estudio contó con el apoyo técnico y financiero del Proyecto Vigía "Enfrentando las Amenazas de las Enfermedades Infecciosas Emergentes y Reemergentes" (Minsa/ Usaid), a través del IV Concurso Nacional para el Desarrollo de Estudios de Investigación en Enfermedades Infecciosas Emergentes y Reemergentes - Año 2003.

\section{REFERENCIAS BIBLIOGRÁFICAS}

1. Randhawa HS. Respiratory and systemic mycoses: an overview. Indian J Chest Dis Allied Sci. 2000;42(4):207219.

2. Rippon JW. Tratado de Micología Médica, Hongos y Actinomicetos patógenos. $3^{\mathrm{a}}$ ed. Buenos Aires: Interamericana, MacGraw Hill; 1990.

3. Valdéz H, Salata RA. Bat-associated histoplasmosis in returning travelers: case presentation and description of a cluster. J Travel Med. 1999;6(4):258-260.

4. Olalla J, Ramón Costa J, Rioperez E, Yubero B. Histoplasmosis pulmonar aguda difusa tras un viaje a Perú. Med Clin (Barc). 2001;116(17):679.

5. García R, Ugarte G, Vidotto V. Paracoccidioidomycosis in Quillabamba, Peru, and its surroundings. Mycoses. 1991;34(11-12):493-497.

6. Arce A, Guillermo J, Torres J, Casquero J. Aspergiloma pulmonar en el Hospital de Apoyo Departamental de Ica, Perú. 2000-2001. Rev Peru Med Exp Salud Publica. 2002;19(4):197-201.

7. Casquero J, Demarini J, Castillo, M. Candella, J. Zurita, S. Nuevos casos de paracoccidioidomicosis. Rev Med Exp. 1998;15(1-2):35-38.

8. Zurita S, Sánchez E, Casquero J. Reporte de caso de aspergiloma. En: Libro de Resúmenes del I Congreso Internacional de Biología. Lima: Sociedad Peruana de Biología; 1999. p. II.26. 
9. Pesce H. Blastomicosis sudamericana: bibliografía y casuística peruana; recopilación y ordenación cronológica. Rev Per Tub Enf Resp. 1965; 25:196-202.

10. Burstein Z. Aporte al diagnóstico de las micosis humanas en el Perú. [Tesis de Doctorado]. Lima: Facultad de Medicina, Universidad Nacional Mayor de San Marcos; 1970.

11. Romero O, Burstein Z. Blastomicosis sudamericana en el Perú. En: Memoria del VII Congreso Ibero Latinoamericano de Dermatología, Caracas, 1971. Caracas: Ed. Síntesis Dos Mil; 1972: 864-72.

12. Mayorca F. Blastomicosis sudamericana pulmonar, contribución a su estudio en el Perú. En: X Congreso Nacional de Tuberculosis y Enfermedades Respiratorias. Tacna: Sociedad Peruana de Tisiología y Enfermedades Respiratorias; 1972: 481-88.

13. Canteros C, Davel G, Rodero L. Inmunodiagnóstico de las micosis sistémicas. En: El laboratorio y el diagnóstico de las micosis sistémicas. Buenos Aires: Instituto de Enfermedades Infecciosas ANLIS «Carlos G. Malbrán»; 2001: 99-108.

14. Cermeño JR, Cermeño JJ, Hernández I, Godoy G, Cermeño $\mathrm{JJ}$, Cabello I, et al. Histoplamine and paracoccidiodine epidemiological study in Upata, Bolívar state, Venezuela. Trop Med Intern Health. 2005;10(3):216-219.

15. Kalmar E, Alencar F, Alves P, Pang W, Del Negro G, Camargo Z, et al. Paracoccidioidomycosis: an epidemiologic survey in a pediatric population from the brazilian amazon using skin tests. Am J Trop Med Hyg. 2004;71(1):82-86.

16. Bueno JP, Mendes-Giannini MJ, Del Negro GM, Assis CM, Takiguti CK, Shikanai-Yasuda MA. Ig G, Ig M and Ig A paracoccidioidomycosis: comparison of counterimmunoelectrophoresis and complement fixation. J Med Vet Mycol. 1997;35:213-217.

17. Canteros C, Rivas M, Soria W, Lee D, Perrotta L, Rodero $\mathrm{G}$, et al. Inmunodiagnóstico de micosis endémicas y aspergilosis broncopulmonar: estudio multicéntrico en la Republica Argentina. Rev Arg Microbiol. 2004;36(2):6874.

18. Hamilton AJ. Serodiagnosis of histoplasmosis, paracoccidioidomycosis and penicilliosis marneffei; current status and future trends. Med Mycol. 1998;36(6):351-364.

19. Faux JA, Shale DJ, Lane DJ. Precipitins and specific Ig G antibody to Aspergillus fumigatus in a chest unit population. Thorax. 1992;47:48-52.

20. Ferreira-Da-Cruz MF, Wanke B, Primes C, Galvao-Castro B. Aspergillus fumigatus fungus ball in hospitalized patients with chronic pulmonary disease. Usefulness of double immunodiffusion test as a screening procedure. Mem Inst Oswaldo Cruz. 1988;83:357-360.

21. Zurita S, Casquero J. Manual de procedimientos de laboratorio para el diagnóstico de micosis oportunista y profunda. Lima: Instituto Nacional de Salud; 1997. Serie de Normas Técnicas $\mathrm{N}^{\circ} 23$.

22. Burstein Z. Aspectos clínicos de la blastomicosis sudamericana (Paracoccidioidomicosis) en el Perú. Rev Peru Med Exp Salud Pública. 2002;19(1):43-47.

23. Severo L, Oliveira F, Irion K, Porto N, Londero A. Histoplasmosis in Rio Grande do Sul, Brazil: a 21-year experience. Rev Inst Med Trop S Paulo. 2001;43(4):18347.

24. Rubinstein A, Negroni R. Micosis broncopulmonares del adulto y del niño. Buenos Aires: Ed. Beta; 1981.

25. Walsh TJ. Recent progress and current problems in treatment of invasive fungal infections in neutropenic patients. Infect Dis Clin North Am. 1996;10(2):365-400.

26. Gassiot-Nuno C. A propósito de las micosis pulmonares. Acta Med. 2000;9(1-2):59-66.

27. Kuan-Yu Ch, Shiann-Chin K, Po-Ren H, Kwen-Tay L, Pan-Chyr Y. Emphasis on microbiological spectra, patient outcome, and prognostic factors. Chest. 2001;120(1):177184.

28. Chadeganipour M, Shadzi S, Dehghan P, Bijary J. The incidence of opportunistic fungi in patients suspected of tuberculosis. Mycoses. 2000;43:269-272.

29. Negroni P, Negroni R. Micosis cutáneas y viscerales. $7^{\text {a }}$ ed. Buenos Aires: López Libreros ed.; 1980.

30. Dourojeanni MJ, Tovar A. Notas sobre el ecosistema y la conservación de la Cueva de las Lechuzas, Parque Nacional de Tingo María, Perú. Rev Forestal Perú (Lima). 1972;5:2845.

Manuscrito recibido el 5 de junio de 2006 y aceptado para publicación el 28 junio de 2006.

\section{Correspondencia:}

Dr. Walter Gómez Gonzáles

Hospital Tingo María - EsSalud.

Jr. Bolognesi $N^{\circ} 120$, Rupa Rupa.

Ниаписо, Perú

Correo-e: waltergomez29@yahoo.com epidemio.tmaria@essalud.gob.pe 